DOI: $10.17234 / S R A Z .65 .20$

UDK: 821.133.1.09 Delerm, Ph. UDK: 821.133.1.09 Adam, O.

Original scientific paper

Reçu le 30 avril 2020

Accepté pour la publication le 25 novembre 2020

\title{
Les petits pois et le platane : deux visages du minimalisme français
}

\author{
Marinko Koščec \\ Université de Zagreb \\ Faculté de Philosophie et Lettres \\ mkoscec@ffzg.hr
}

L'étude juxtapose deux recueils de textes brefs publiés vers le tournant du siècle, La Première Gorgée de bière, et autres plaisirs minuscules de Philippe Delerm, et Passer l'hiver d'Olivier Adam. En dépit de la divergence radicale de leurs prémisses, visées et poétiques, notre analyse dévoile certains aspects que ces deux énoncés partagent, notamment l'appartenance à la veine minimaliste. L'étude démontre comment les écritures de Delerm et d'Adam, chacune de sa manière authentique, s'approchent et s'écartent de différentes versions du minimalisme, dont elles incarnent deux figures antipodiques.

Mots-clés : littérature, français, minimalisme, Delerm, Adam

La Première Gorgée de bière et autres plaisirs minuscules de Philippe Delerm (1997) et Passer l'hiver d'Olivier Adam (2004), recueils de textes brefs emblématiques de la production de leur auteur, figurent parmi les rares exceptions à la règle selon laquelle, dans le paysage de la prose contemporaine en français, seul le genre romanesque permet «la visibilité » : les deux ont été très favorablement reçus par le public comme par la critique. Bien qu'à premier abord ils révèlent des moyens et des objectifs radicalement divergents, un regard plus attentif dévoilera des aspects où ces deux énoncés se rejoignent, notamment leur appartenance à la famille minimaliste.

Le succès de La Première gorgée de bière lui a valu le statut de l'ouvrage paradigmatique de toute une tendance littéraire. ${ }^{1}$ Il ne s'agirait pas d'un mouvement, mais d'une sensibilité commune, d'une famille d'esprit aspirant à revaloriser l'existence journalière et à débanaliser l'ordinaire. À partir d'environ 1990, cette veine littéraire fournit un antidote à l'ultramoderne, à la tyrannie du spectacle et du zapping comme manière de vivre et penser. Avec patience

1 Dans les années 90 du siècle dernier, la critique a rassemblé quelques prosateurs sous des étiquettes comme « les moins que rien » ou « les chroniqueurs du peu ». Voir par exemple Rivage (1998 : 8-9) ou Arambasin (2007 : 56). 
et humilité, cette littérature raconte la France profonde, de terroir, celle de la tradition et de la vie familiale. La seule " aventure » y est celle de l'homme domestique qui se consacre à son intimité et médite sur ce qui constitue son entourage humain et surtout naturel. Chez Delerm en particulier, ce parti pris éthique et esthétique débouche sur un «minimalisme positif » (Bertrand 2005), proposant un contrepoids au scepticisme, au manque de repères et au vide intérieur, que l'auteur cherche à surmonter par la célébration du quotidien et de ses richesses discrètes.

Également ancrées dans vie quotidienne, les nouvelles d'Adam suivent des gens qu' on appelle ordinaires dans leurs maisons et appartements de la banlieue, dans les bureaux, couloirs d'hôpitaux, supermarchés. Comme chez Delerm, l'intrigue est minimisée, voire annulée, mais la tension narrative et le suspense sont sensibles. L'ellipse y est un ressort important de la stratégie narrative ; les personnages de Passer l'hiver montrent des empreintes de différents traumatismes, mais la déchirure et le deuil ne sont qu'évoqués, esquissés par une main laconique. Bien qu'on soit sans cesse au bord d'un précipice ou d'une explosion, le drame n'éclate jamais ; l'événement reste en dehors de la tranche de vie représentée.

Le minimalisme littéraire n'entend pas une formule unique mais une pluralité de principes et stratégies. En général, il s'agit d'éviter le modèle balzacien du réalisme psychologique et de résister au dispositif narratif convenu qui suppose la complétude. Or, si la variation que Thibault (2012 : 121) appelle « le récit minimaliste » se caractérise surtout par la mise en scène insistante des réticences du narrateur par rapport à sa propre activité diégétique, nos deux auteurs relèvent plutôt du « récit minimal », qui présente des actions peu nombreuses ou décrit de menus événements sans conséquences, donc un « minimum vital » de la fiction narrative. Bien que chez Delerm comme chez Adam on ne trouve pas de véritables événements, le récit est clairement là, même si celui-ci peut susciter le sentiment d'un "véritable enlisement », faute de toute " téléologie événementielle » ou «vectorisation du texte vers son dénouement » (Audet). De même, nos auteurs partagent la parenté avec « l'écriture blanche »- dans la mesure où celle-ci présente une histoire comme si elle en était complètement désinvestie, avec un degré zéro de la rhétorique (Rabaté et Viart 2009: 190) - mais sans miser sur le non-dit ou un certain artisme au niveau langagier. La prose delermienne est cependant plus proche de ce que Richard (1999: 217) a nommé «essai-poème». Tissés essentiellement d'éléments descriptifs et tournures itératives, ces textes représentent chacun une situation unique, avec une succession d'états, donc un parcours bref de sensations ; si une action y survient, elle ne se développe jamais en intrigue. ${ }^{2}$ En principe, la mise en récit du quotidien suppose le surgissement d'un élément insolite ou inattendu ; son absence peut conduire à considérer le récit comme déficient. ${ }^{3}$ En peignant la vie

2 Ceci les approche également de ce que Godenne appelle la «nouvelle-instant », pour l'opposer à la « nouvelle-histoire » (Godenne 1995 : 110).

3 Comme le souligne Audet, « l'attente de l'inattendu dans le récit s'inscrit difficilement dans l'exercice de la peinture de la vie courante, qui se caractérise justement par l'absence de singularités ou de faits marquants» (op. cit.). 
ordinaire, Delerm met en avant précisément son caractère répétitif, routinier, donc l'absence de moments saillants. ${ }^{4}$ Le seul événement de sa prose est la mutation de l'ordinaire en extraordinaire grâce à une alchimie poétique qui tend à transformer le moindre fait en expérience presque mystique.

Déjà les titres de la plupart des 34 textes brefs qui composent le recueil permettent de deviner le rejet de tout ce qui est spectaculaire et dramatique, voire de l'événement en tant que fondement du récit : Le paquet de gâteaux du dimanche matin, Prendre un porto, L'odeur des pommes, Aller aux mûres, etc. Il s'agit plutôt de focaliser des détails de l'existence que Perec (1989) a appelés infra-ordinaires ou endotiques : objets ou gestes généralement considérés comme triviaux et insignifiants. Le légume évoqué dans le titre de notre étude se trouve dans la nouvelle intitulée Aider à écosser les petits pois ; comme dans les autres, le procédé fondamental y est l'inversion souriante de ce qu' on prend habituellement pour la manifestation de la pure banalité. De tels motifs sont systématiquement abordés par un discours apparemment humble, puis grossis au point d'obtenir une valeur poétique, voire une aura métaphysique ou allégorique. Hyperbolisée, cette « action » anodine, voire infantile, devient une métaphore du bonheur domestique et familial. Sous le masque d'une réduction, il s'agit donc d'une amplification et d'une sublimation. L'ensemble compose une rhétorique apaisante et sereine de la simplicité, de la lisibilité immédiate du monde, autrement dit une éthique de l'immanence, du présent et du contentement.

Chez Adam, au contraire, on trouvera une tonalité sombre, la permanence de l'angoisse et du regret ; la face noire du minimalisme positif. Son quotidien est banal et impassible : la grisaille et la monotonie de la périphérie des villes, de ceux qui restent en marge de la société de l'aisance et de la consommation festive. Également sensible aux menus détails de la vie, cet auteur guette et raconte des mouvements, mais davantage encore il saisit les ambiances, en insistant sur les temps morts, les creux, le failles et les absences. Si Delerm amplifie et glorifie presque rien, Adam dit presque rien sur des choses immenses, trop grandes pour les mots, telles que la mort, le désespoir, le vide. Les nouvelles représentent des vies sur le seuil de l'écroulement, marquées par l'insécurité, la solitude ou la perte de quelqu'un ou quelque chose (la fin d'une histoire d'amour, la disparition du désir, la mort d'un être cher, etc.). Ce qui rassemble les sujets de ces différentes crises est leur résignation face à la fatalité. D'une part, ils sont tous victimes de l'exclusion sociale, mais au-delà de toute condition extérieure, ils partagent aussi une conscience aigüe de la mort, de la déperdition consubstantielle à la vie. La note commune et le point final de toutes ces nouvelles, c'est le vide, sans réconfort ni espoir.

En revanche, chez Delerm, comme chez les mystiques, le vide (donc l'absence de la grande émotion, voire de tout événement) donne l'accès à la plénitude. $\mathrm{Au}$ lieu de se résigner au malheur, le sujet delermien se contente pleinement de son existence et de sa personne. En se débarrassant de l'espérance, il aboutit à

4 On pourrait donc y utiliser bien à propos le terme « anodal », que Viegnes (2014:14) attribue au récit bref où la narrativité est totalement ou presque absente. 
un bonheur inespéré et désespéré. Ce désespoir positif est donc le résultat d'un travail: de la volonté d'abandonner ce qu'on n'a pas et de désirer ce qu'on possède. La vie ainsi présentée devient un paradis terrestre, où la joie cohabite avec la souffrance, la plénitude de l'instant avec la mort. Par cela même, cette doctrine s'approche au stoïcisme, car le sage stoïcien reçoit sereinement ce qu'il ne peut pas changer. Or, au lieu de la pure impassibilité et de l'indifférence, le stoïcisme delermien se colore de l'étonnement permanent qui l'approche à l'épicurisme, dans la mesure où celui-ci écarte tout discours métaphysique réduisant la vie à l'antichambre de la véritable existence, et n'envisage pas d'autre récompense que le plaisir qu'on peut tirer de la vie. De même, les appels à ne rechercher que ce qui se trouve dans la nature apparentent cet énoncé au naturalisme, voire à une sorte d'écologisme philosophique (qui s'écarte de l'écologisme pragmatique ou politique), revendiquant une véritable fusion entre l'homme et le milieu naturel. Dans le sillon de Rousseau, ce naturaliste épicurien donne des recettes comment vivre conformément à l'atemporelle nature, alors qu'implicitement il dénonce l'aliénation et les plaisirs décadents qui proviennent de la société.

Or, cette apologie du retour au sein de la nature et à l'unité prélogique avec l'univers attribue la priorité aux sensations au point de rejeter la raison. Dans le minimalisme delermien, la pensée naît de la sensation. Ce n'est que par les sens, surtout le regard, qu'on accède au réel, et par cela-même à la valeur de la vie : le sujet créé de la beauté par son regard. En revanche, toute considération rationnelle devient secondaire, superflue, voire une injure à la beauté. Cette lecture du monde conduit ainsi à sa dérationalisation, au nom de la plénitude sensuelle, ce qui pourrait être interprété comme une version du dépassement bouddhiste de l'individu : le moi du sujet poétique delermien subsiste, mais comme allégé, purifié, libéré. En plongeant dans le monde, il s'oublie, s'arrache à son orgueil et à sa solitude.

À l'antipode, les nouvelles de Passer l'hiver sont des drames du sujet, théâtrales et tragiques. Les personnages sont prisonniers et victimes de leur situation individuelle, toujours délicate et pénible. La vie leur a posé des pièges ; elle n'a pas tenu ses promesses et les a abandonnés. Abusés par la société, éloignés mentalement et émotivement de leurs proches, ils sont désenchantés et épuisés, enlisés dans les sables mouvants du quotidien, privés de leur place dans ce monde, « comme des papillons épinglés dont les ailes frémissent encore et qui rêvent de voler, de s'évader » (Adam 2004 : 79).

Alors que ces nouvelles découlent de l'ambiance hivernale et nocturne, les textes de Delerm sont plutôt estivaux et ensoleillés. Ces derniers relèvent d'un temps itératif, car ils focalisent soit un geste fréquent, soit une occurrence qui revient tous les ans vers la même date, comme au sommet ou à la fin de l'été (le Tour de France, la cueillette de mûres). Au lieu de mettre en scène des événements avec une durée et une clôture, l'énoncé delermien produit ainsi un effet d'immobilité et semble flotter dans l'atemporalité.

Chez Adam, au contraire, la temporalité est clairement définie. Chaque nouvelle a son unité de temps : un soir, une nuit entière, une heure particulièrement difficile dans la vie du personnage. La narration commence vers le moment où 
tout pourrait basculer. Dès la première phrase, le lecteur est précipité dans le point de vue des personnages au bord de la crise, par exemple : "Je me sens vide. Tout le temps je pense à ça. Ce vide à l'intérieur. » (47), «En pleine nuit, la lumière blanche, c'était plus froid et cru que jamais » (117). L'auteur y instaure d'emblée la tension et introduit le fantôme de la mort. Or, le recueil possède également une certaine valeur itérative : les nouvelles paraissent comme des variations de la même situation dramatique où une existence entière ne tient qu'à un fil. Cependant, plutôt que de le rompre, Adam laisse le texte à l'état d'attente jusqu'à la fin ; le moment décisif n'arrive pas. C'est donc une autre version de non-événement, du récit qui « déçoit ».

Cela dit, soulignons que l'idée du suicide est toujours dans les parages. Parfois, le désespoir remonte à la surface : "J'ai croisé un semi-remorque, ses phares m'ont aveuglée, j'ai fait un écart et trois secondes ça m'a traversé le crâne, je ne peux pas le nier, ça m'est venu au cerveau, l'idée du platane » (65). Voici donc l'arbre que notre titre prend pour l'effigie de l'approche métonymique d'Adam, fondée sur des euphémismes et des litotes. Le suicide, ici substitué par le platane, reste une pensée passagère. Lorsqu'elle «traverse le crâne », le protagoniste s'accroche à la vie par réflexe ; il s'entête à vivre malgré tout. Le titre du recueil évoque le froid dans les corps et les âmes, mais aussi l'instinct de survie que les protagonistes partagent, leur désir de traverser la période hivernale d'obscurité et de solitude. Passer l'hiver entend à la fois la difficulté, la lourdeur, la résignation, et dans le même temps la volonté de résister, de survivre, fût-ce à soi-même, à son penchant de se laisser couler.

Tout en partageant l'affinité avec des procédés et des visées minimalistes, les deux écritures étudiées présentent des visages antipodiques. Alors que Delerm pratique un art statique, pictural, impressionniste, recherchant avant tout la beauté, celui d'Adam est âpre, acéré et il mise sur des techniques plutôt cinématographiques, perpétuant l'ellipse, le montage «cut » et le refus de la psychologie explicite, qu'il réduit aux gestes et dialogues, et tout cela surtout pour dire le mal d'être. Si l'humanisme de Delerm est éclatant, celui d'Adam transparaît malgré la façade glaciale : tout en représentant ses personnages tels qu'ils sont au plus bas, sans s'apitoyer ni sombrer dans le pathétique, il montre sa solidarité avec eux, son regard est fraternel. Les deux auteurs se distinguent par la douceur, la modestie, l'élégance et la précision du propos. Chacun de sa manière, ils cultivent le goût du laconisme qui, malgré le souci de la sobriété, rigueur et justesse, ne vire jamais dans la sécheresse ; les deux énoncés sont bien plastiques, ancrés dans les réalités matérielles. En dépit de la fausse naïveté chez l'un, et de l'apparente réduction aux traces extérieures chez l'autre, par le biais du fragment et du détail, les deux auteurs parviennent à toucher le nerf de l'existence humaine. 


\section{Bibliographie}

Adam, Olivier (2004). Passer l'hiver, Paris : Éditions de l'Olivier.

Arambasin, Nella (2007). Littérature contemporaine et histoires de l'art. Récits d'une réévaluation, Genève : Droz.

Audet, René (2007). Fuir le récit pour raconter le quotidien: modulations narratives en prose contemporaine, < https:// tempszero.contemporain.info/document $84>$ $(12 / 9 / 2019)$.

Bertrand, Rémi (2005). Philippe Delerm et le minimalisme positif, Paris : Éditions du Rocher.

Delerm, Philippe (1997). La Première Gorgée de bière et autres plaisirs minuscules, Paris : L'Arpenteur.

Godenne, René (1995). La nouvelle, Paris : Honoré Champion.

Perec, Georges (1989). L'infra-ordinaire. Paris : Seuil.

Prince, Gerald (2012). Récit minimal et la narrativité, in : Le récit minimal. Du minime au minimalisme [éd. Sabrinelle Bedrane, Françoise Revaz, Michel Viegnes], Paris : Presses Sorbonne nouvelle.

Rabaté, Dominique et Viart, Dominique [éd.] (2009). Ecritures blanches, SaintÉtienne : Publications de l'Université de Saint-Etienne.

Richard, Jean-Pierre (1999). Essais de critique buissonière, Paris : Gallimard.

Rivage, Bertrand (1998). Les moins que rien, in : La Nouvelle Revue Française, Paris, n540, pp. 8-19.

Thibault, Bruno (2012). Les bâtons rompus de l'écriture : 'l'histoire brisée' entre récit minimal et récit minimaliste, in : Le récit minimal. Du minime au minimalisme. [éd. Sabrinelle Bedrane, Françoise

Revaz, Michel Viegnes], Paris : Presses Sorbonne nouvelle.

Viegnes, Michel (2014). L'œuvre au bref: la nouvelle de langue française depuis 1900, Genève : Baconnière.

\section{Grašak i platana: dva lica francuskog minimalizma}

Ova komparativna analiza zbirki kratkih proznih tekstova La Première Gorgée de bière et autres plaisirs minuscules Philippea Delerma i Passer l'hiver Oliviera Adama razmatra aspekte koji ih povezuju unatoč različitim svjetonazorima i poetikama. Dok se u svojim lirsko-meditativnim fragmentima Delerm fokusira na tzv. endotičke elemente, tj. predmete i geste iz svakodnevnice u kojima otkriva neslućeni alegorijski i/ili metafizički potencijal, odnosno izvore životne radosti, Adamove su novele utemeljene na egzistencijskim dramama te prožete prisutnošću smrti. Studija ipak otkriva različite minimalističke postupke zajedničke ovim pismima, koja bi se stoga mogla smatrati dvama oprečnim licima minimalizma.

Ključne riječi : književnost, francuski, minimalizam, Delerm, Adam 